\title{
The dissipation of the system and the atom in two-photon Jaynes-Cummings model with degenerate atomic levels
}

\author{
Y. Q. Guo, L. Zhou, H. S. Song \\ Department of Physics, Dalian University of Technology, \\ Dalian 116024, China
}

September 22, 2018

\begin{abstract}
The method of perturbative expansion of master equation is employed to study the dissipative properties of system and of atom in the twophoton Jaynes-Cummings model (JCM) with degenerate atomic levels. The numerical results show that the degeneracy of atomic levels prolongs the period of entanglement between the atom and the field. The asymptotic value of atomic linear entropy is apparently increased by the degeneration. The amplitude of local entanglement and disentanglement is suppressed. The better the initial coherence property of the degenerate atom, the larger the coherence loss.
\end{abstract}

PACS: 42.50.Pq, 42.50.-p, 03.67.-a

\section{Introduction}

A main obstacle of quantum information is the decoherence of qubits. A quantum system inevitably interacts with its surrounding environment, and the interaction between the quantum system and its surrounding could lead to decoherence. Therefore, studying decoherence process [1, 2, 3] and solving the problem 4] have attracted much attention. Many authors stressed that the decoherence is induced by the role of environment dissipation [1, 2, 3].

As a candidate of quantum information, cavity QED has been widely studied in follow aspects: quantum network [5, 6], producing entanglement [7, 10] and 
quantum teleportation [8, 9, 10, especially in the dissipative dynamics 2, 3, 11]. This is because: the dissipation of cavity QED could be monitored in experiment 2, 11, and this is valuable not only to the candidate of quantum information but also to the research of decoherence theory. In Ref. [3], the dissipative dynamics of the JCM in the dispersive approximation has been studied, and the influence of dissipation on the entanglement of the two subsystems was investigated. In Ref. 12, the authors studied the field state dissipative dynamics of two-photon JCM and showed that the cavity dissipation affected the coherence properties of the field. In these studies, the atom is a single pure two-level one. While, in some case, atomic level is degenerate in the projections of the angular momenta on the quantization axis [13]. In Ref. [14, 15], the authors studied the case of degenerate atomic levels and showed that the revival period of the atom population inversion became longer than that of the original JCM. In Ref. [16], our group has studied the effect of degenerate atomic levels on the field state dissipation in two-photon JCM. The initial degenerate atomic states are found to increase the period of entanglement of atom and field, and the coherence properties of field is affected by the reservior qualitatively.

The interaction between an atom and a single-mode field via two-photon process is a nonlinear one. Giving the explicit solution of the dissipative dynamics of atom in a nonlinear process is very difficulty. So, the atomic decoherence property in a two-photon JCM with degenerate atomic levels is not studied before, despite that the field decoherence property has been studied [12, 16]. However, the atom is the information depositor in a quantum information system [17; it is more important to study the atomic decoherence property than that of the field. In the present paper, considering degenerate atomic levels and using the method of perturbative expansion for master equation, we study the dissipative dynamics of system and of atom in two-photon JCM. We show that the period of entanglement between atom and field is prolonged explicitly. In 
case of degenerate atomic levels, contrasting to the case of two levels atom, the atomic asymptotic value of linear entropy is increased clearly in a same dissipative cavity. This asymptotic value is the largest when the atom is initially in an equal probability superposition degenerate state. Moreover, the numerical results show that the degenerate atomic levels increase the mixture degree of nonlinear situation of two-photon JCM.

\section{Perturbative solution of master equation}

Let us consider an atom with degenerated levels interacts with a single mode electromagnetic field at zero temperature as usual in a high-Q cavity. The dynamics of the global density operator $\hat{\rho}$ in interaction representation is governed by the master equation

$$
\frac{d}{d t} \hat{\rho}=-i\left[H_{e f f}, \hat{\rho}(t)\right]+\kappa\left(2 a \hat{\rho} a^{\dagger}-a a^{\dagger} \hat{\rho}-\hat{\rho} a a^{\dagger}\right)
$$

where $\kappa$ is the damping constant of the cavity. The effective Hamiltonian $H_{e f f}$ in two-photon JCM with atomic degeneracy is nonlinear. Thus Eq. (1) is a nonlinear differential equation. Completely solving the equation with the usual method used in the study of original JCM is very difficult. In order to study the dissipative dynamics of two-photon JCM, we can solve the master equation approximately [18]. The $\hat{\rho}(t)$ can be expanded in powers of $\kappa$ as

$$
\hat{\rho}(t)=\hat{\rho}^{0}(t)+\frac{\partial \hat{\rho}(t)}{\partial \kappa} \kappa+\frac{1}{2} \frac{\partial^{2} \hat{\rho}(t)}{\partial \kappa^{2}} \kappa^{2}+0\left(\kappa^{2}\right) .
$$

In a high-Q cavity, $\kappa \gg 1$, the effectiveness of the expansion is assured and the reservation of only fore 3 terms is satisfying.

From Eq. (1), each orders of differential of $\hat{\rho}(t)$ can be determined by using 
iterative method from Eq. (1) as

$$
\frac{d}{d t} \hat{\rho}^{(n)}(t)=-i\left[\hat{H}_{e f f}, \hat{\rho}^{(n)}(t)\right]+\left(2 a \hat{\rho}^{(n-1)}(t) a^{\dagger}-a a^{\dagger} \hat{\rho}^{(n-1)}(t)-\hat{\rho}^{(n-1)}(t) a a^{\dagger}\right),
$$

with $n=1,2,3 \ldots$,and $\hat{\rho}^{(n)}(t)=\frac{\partial^{n} \rho(t)}{\partial \kappa^{n}}$. The solution of Eq. (3) is

$$
\begin{aligned}
\hat{\rho}^{(n)}(t)= & e^{-i \hat{H}_{e f f} t}\left\{\int _ { 0 } ^ { t } d t e ^ { i \hat { H } _ { e f f } t } \left[2 a \hat{\rho}^{(n-1)}(t) a^{\dagger}-a a^{\dagger} \hat{\rho}^{(n-1)}(t)-\hat{\rho}^{(n-1)}(t)\right.\right. \\
& \left.\left.\left.a a^{\dagger}\right)\right] e^{-i \hat{H}_{e f f} t}\right\} e^{i \hat{H}_{e f f} t} .
\end{aligned}
$$

So, $\hat{\rho}^{(1)}(t)$ can be calculated from an integer of $\hat{\rho}^{(0)}(t)$, and $\hat{\rho}^{(2)}(t)$ from $\hat{\rho}^{(1)}(t)$, ... . Thus we can get arbitrary value of $\hat{\rho}(t)$ at least in principle.

We assume that the initial states of the atom-field system can be written as

$$
\Psi(0)=\left(\sum_{m_{b}} \frac{e}{\sqrt{2 J_{b}+1}}\left|J_{b}, m_{b}\right\rangle+\sum_{m_{c}} \frac{f}{\sqrt{2 J_{c}+1}}\left|J_{c}, m_{c}\right\rangle\right) \otimes|\alpha\rangle,
$$

where $J_{b}, J_{c}$ denote the values of the total electronic angular momenta of resonant levels $b, c$ respectively, and $m_{b}, m_{c}$ represent their projections on the polarized direction. $|\alpha\rangle$ is the coherence field state of cavity; it can be expanded as $|\alpha\rangle=\exp \left(-\frac{|\alpha|^{2}}{2}\right) \sum_{n} \frac{\alpha}{\sqrt{n !}}|n\rangle=\sum_{n} F_{n}|\alpha\rangle$. From Eq. (5), we easily obtain the initial density of the system, i.e. $\hat{\rho}^{(0)}(0)$, and further obtain the evolution of $\hat{\rho}^{(0)}(0)$. In interaction representation, the effective Hamiltonian of the twophoton JCM within rotating-wave approximation including degenerate atomic levels has been obtained in Ref. [16] as

$$
\hat{H}_{e f f}=\Omega\left[\left(a^{\dagger} a+1\right)\left(a^{\dagger} a+2\right) R_{b}-\left(a^{\dagger} a-1\right) R_{c}\right],
$$

where $R_{\mu}=\sum_{m \mu} \alpha_{m \mu}^{2}\left(\left|J_{\mu}, m_{\mu}\right\rangle\left\langle J_{\mu}, m_{\mu}\right|\right), \mu=b, c . \Omega$ is the Rabi frequency which measures the coupling between atom and field. $\alpha_{m \mu}$ is defined as $\alpha_{m \mu}=$ $(-1)^{J_{b}-m}\left(\begin{array}{lll}J_{b} & 1 & J_{c} \\ -m & 0 & m\end{array}\right)$.

After some deducing, we obtain the density matrix of the system as 


$$
\begin{aligned}
\hat{\rho}(t)= & \sum_{n, n^{\prime}, m, m^{\prime}} F_{n} F_{n^{\prime}}^{*}\left\{\exp \left\{-i \Omega t\left[(n+1)(n+2) \alpha_{m}^{2}-\left(n^{\prime}+1\right)\left(n^{\prime}+2\right) \alpha_{m^{\prime}}^{2}\right]\right\}\right. \\
& \frac{e^{2}}{2 J_{b}+1} A_{b b n n^{\prime} m m^{\prime}}\left|n, J_{b}, m\right\rangle\left\langle n^{\prime}, J_{b}, m^{\prime}\right|+\exp \left\{i \Omega t \left[n(n-1) \alpha_{m}^{2}-\right.\right. \\
& \left.\left.n^{\prime}\left(n^{\prime}-1\right) \alpha_{m^{\prime}}^{2}\right]\right\} \frac{f^{2}}{2 J_{c}+1} A_{c c n n^{\prime} m m^{\prime}}\left|n, J_{c}, m\right\rangle\left\langle n^{\prime}, J_{c}, m^{\prime}\right|+\exp \{-i \Omega t \\
& {\left.\left[(n+1)(n+2) \alpha_{m}^{2}+n^{\prime}\left(n^{\prime}-1\right) \alpha_{m^{\prime}}^{2}\right]\right\} \frac{e f}{\sqrt{\left(2 J_{b}+1\right)\left(2 J_{c}+1\right)}} B_{b c n n^{\prime} m m^{\prime}} } \\
& \left|n, J_{b}, m\right\rangle\left\langle n^{\prime}, J_{c}, m^{\prime}\right|+\exp \left\{i \Omega t \left[n(n-1) \alpha_{m}^{2}+\left(n^{\prime}+1\right)\left(n^{\prime}+2\right) \alpha_{m^{\prime}}^{2}\right.\right. \\
& ]\} \frac{e f}{\sqrt{\left(2 J_{b}+1\right)\left(2 J_{c}+1\right)}} B_{b c n n^{\prime} m m^{\prime}}^{*}\left|n, J_{c}, m\right\rangle\left\langle n^{\prime}, J_{b}, m^{\prime}\right|,
\end{aligned}
$$

where we have set

$$
\begin{aligned}
A_{\mu \mu n n^{\prime} m m^{\prime}}= & 1+\frac{\kappa}{\Omega}\left[ \pm i N F\left(n, n^{\prime}, m, m^{\prime}, t\right)-\left(n+n^{\prime}\right) \Omega t\right]+\frac{1}{2}\left(\frac{\kappa}{\Omega}\right)^{2}\{ \\
& \frac{N^{2}}{n \alpha_{m}^{2}-n^{\prime} \alpha_{m^{\prime}}^{2}}\left[F\left(n, n^{\prime}, m, m^{\prime}, t\right)-\frac{1}{2} F\left(n, n^{\prime}, m, m^{\prime}, 2 t\right)\right]- \\
& N\left[\frac{F\left(n, n^{\prime}, m, m^{\prime}, t\right)}{n \alpha_{m}^{2}-n^{\prime} \alpha_{m^{\prime}}^{2}} \pm i\left(n+n^{\prime}+2\right) F\left(n, n^{\prime}, m, m^{\prime}, t\right) \Omega t \pm\right. \\
& \left.\left.\frac{i 2 \Omega t}{n \alpha_{m}^{2}-n^{\prime} \alpha_{m^{\prime}}^{2}}\right]+\frac{\left(n+n^{\prime}\right)^{2}}{2}(\Omega t)^{2}\right\}, \\
B_{b c n n^{\prime} m m^{\prime}}= & 1+\frac{\kappa}{\Omega}\left[i N E\left(n, n^{\prime}, m, m^{\prime}, t\right)-\left(n+n^{\prime}\right) \Omega t\right]+\frac{1}{2}\left(\frac{\kappa}{\Omega}\right)^{2}\{ \\
& \frac{N^{2}}{(n+3) \alpha_{m}^{2}+\left(n^{\prime}+1\right) \alpha_{m^{\prime}}^{2}}\left[E\left(n, n^{\prime}, m, m^{\prime}, t\right)-E\left(n+\frac{1}{2},\right.\right. \\
& \left.\left.n^{\prime}+\frac{1}{2}, m, m^{\prime}, 2 t\right)\right]-N\left[\frac{E\left(n, n^{\prime}, m, m^{\prime}, t\right)}{(n+2) \alpha_{m}^{2}+n^{\prime} \alpha_{m^{\prime}}^{2}}+\left(n+n^{\prime}+2\right)\right. \\
& \left.i \Omega t E\left(n, n^{\prime}, m, m^{\prime}, t\right)+\frac{i 2 \Omega t}{(n+2) \alpha_{m}^{2}+n^{\prime} \alpha_{m^{\prime}}^{2}}\right]+\frac{1}{2}\left(n+n^{\prime}\right)^{2}(\Omega t)^{2},
\end{aligned}
$$

with

$$
\begin{gathered}
F\left(n, n^{\prime}, m, m^{\prime}, t\right)=\frac{\exp \left\{-i 2 \Omega t\left(n \alpha_{m}^{2}-n^{\prime} \alpha_{m^{\prime}}^{2}\right)\right\}-1}{n \alpha_{m}^{2}-n^{\prime} \alpha_{m^{\prime}}^{2}}, \\
E\left(n, n^{\prime}, m, m^{\prime}, t\right)=\frac{\exp \left\{-i 2 \Omega t\left[(n+2) \alpha_{m}^{2}+n^{\prime} \alpha_{m^{\prime}}^{2}\right]\right\}-1}{(n+2) \alpha_{m}^{2}+n^{\prime} \alpha_{m^{\prime}}^{2}},
\end{gathered}
$$


$N=|\alpha|^{2}$, and,$+(-)$ is chosen from \pm when $\mu=b, c$ respectively.

Now,taking the trace of $\hat{\rho}(t)$ on field states variables, we can obtain the reduced atomic density

$$
\begin{aligned}
\hat{\rho}_{a}(t)= & \sum_{m, m^{\prime}, n, n} F_{n} F_{n}^{*}\left\{\frac{e^{2}}{2 J_{b}+1} A_{b b n n m m^{\prime}}\left|J_{b}, m\right\rangle\left\langle J_{b}, m^{\prime}\right|+\frac{f^{2}}{2 J_{c}+1} A_{c c n n m m^{\prime}}\right. \\
& \left|J_{c}, m\right\rangle\left\langle J_{c}, m^{\prime}\right|+\exp \left\{-i \Omega t\left[(n+1)(n+2) \alpha_{m}^{2}+n(n-1) \alpha_{m^{\prime}}^{2}\right]\right\} \\
& \frac{e f}{\sqrt{\left(2 J_{b}+1\right)\left(2 J_{c}+1\right)}} B_{b c n n m m^{\prime}}\left|J_{b}, m\right\rangle\left\langle J_{c}, m^{\prime}\right|+\exp \{i \Omega t[n(n-1) \\
& \left.\left.\alpha_{m}^{2}+(n+1)(n+2) \alpha_{m^{\prime}}^{2}\right]\right\} \frac{e f}{\sqrt{\left(2 J_{b}+1\right)\left(2 J_{c}+1\right)}} B_{b c n n m m^{\prime}}^{*}\left|J_{c}, m\right\rangle \\
& \left\langle J_{b}, m^{\prime}\right| .
\end{aligned}
$$

The linear entropy is defined as

$$
S=1-\operatorname{Tr}\left(\hat{\rho}^{2}\right)
$$

which can be used to measure the coherence lose and the purity degree of the state. For a pure state $S=0$, otherwise, $S>0$, corresponding to a mixture.

Substitute $\hat{\rho}(t), \hat{\rho}_{a}(t)$ into Eq. (9). We get the linear entropy of system $S$ and of atom $S_{d a}$. Here, we use letter $d$ to denote atomic linear entropy in JCM in case of degenerate atomic levels. The linear entropy $S_{d a}$ then depends on a set of parameters $\left(e, f,|\alpha|^{2}, J_{b}, J_{c}, \frac{\kappa}{\Omega}, \Omega t\right)$. In next section, typical values of the parameters are given to discuss the behavior of $S, S_{d a}$, and from the appearance of the linear entropy, the decoherence of the system and the atom and the disentanglement of atom and field are studied.

\section{Results and Discussion}

In experiment [19], the resonant atomic levels $b, c$ were usually the Rydberg states of the atom with angular momenta $J_{b}=\frac{3}{2}, J_{c}=\frac{3}{2}$ or $J_{c}=\frac{5}{2}$. By the support of these experiment data, we choose $J_{b}=J_{c}=\frac{3}{2}$, which emerges $\alpha_{\frac{1}{2}}=\alpha_{-\frac{1}{2}}=\frac{1}{2 \sqrt{15}}, \alpha_{\frac{3}{2}}=\alpha_{-\frac{3}{2}}=\frac{3}{2 \sqrt{15}}$. 
With same set of parameters, we plot the evolution of atomic linear entropy with and without degeneration to study the effect of atomic degeneracy on the dissipative dynamics of atom in Fig.1.

Without degeneration, the atomic is a pure two-level one. Under this case, the linear entropy has been discussed in Ref. [20. We directly employ the result in that paper to show the effect of the atomic degeneracy. In the process of atomic linear entropy evolution, Fig.1 show that the behavior of $S_{a}(t)$ (atomic linear entropy in non-degenerate case) and $S_{d a}$ both present local maximum and minimum. This corresponds to the entanglement and disentanglement respectively of atom and field. In Ref. 3, the authors have shown that disentanglement took place at instants $t_{d}=\frac{n \pi}{\omega}$. Here, contrasting to the case of non-degeneration [20], the period of entanglement and disentanglement in case of degenerate atomic levels is obviously prolonged. And the amplitude of local entanglement and disentanglement is suppressed. We can also observe that the two asymptotic values are obviously different, i.e. $S_{d a}(\infty)>S_{a}(\infty)$. The linear entropy is seen as a measure of the coherence loss. If the atom is initially in a degenerate state written in Eq. (5), the coherence property is better than that of without degeneracy. When coherence loss finished completely, the better the initial coherence property the bigger the coherence loss. We will show the effect of initial coherence property on the coherence loss in Fig.3. Through comparing the atomic linear entropy with and without degeneracy, we find that the degeneracy increases the maximum of the asymptotic value of the linear entropy. Thus, the degeneracy of the atom on one hand increase the period of entanglement on the other hand increases the atomic maximum coherence loss. The result has not been given before.

The dependence of the linear entropy on the photon number is shown in Fig.2. It is clear that the period of entanglement does not depend on the average photon number $|\alpha|^{2}$. And obviously, the larger the average photon number, the 
larger the asymptotic value of the curves. The similar results have been shown in Ref. 3. In that paper, the asymptotic values of the linear entropy never exceed $\frac{1}{2}$, while here, the asymptotic values can do. The average photon number $|\alpha|^{2}$ is a measure of its classicality. The entanglement of atom and field proportionally contributes to $S_{d a}$. Therefore, the larger the $|\alpha|^{2}$ is the more rapidly the atom and the system lose their coherence. These properties are also observed in Ref. [3] 15.

Now, we take different initial atomic degenerate states to show their effect on the disentanglement and decoherence of atom. In Fig.3, when the atom is initially in degenerate state with $e=f=1 / \sqrt{2}$, the asymptotic value is the largest. In this case, the initial coherence of atom is the best; when dissipation finally finishes, the coherence loss is the most and the linear entropy is the largest. Fig. 3 also shows that the amplitude of local maximum and minimum of the curves is the largest in this case. Because of the best initial coherence property of atom, the atom would best entangle with field. So, the better the initial atomic coherence the larger the amplitude of local maximum and minimum. Therefore, the better the initial atomic coherence properties, the larger the asymptotic value of $S_{d a}$ and the larger the amplitude of local maximum and minimum of $S_{d a}$.

\section{Conclusion}

We have studied the dissipative dynamics of system and atom in two-photon JCM with degenerate atomic levels by employing the perturbtive expansion of master equation. The results show that the degeneracy prolongs the period of entanglement and disentanglement of atom and field qualitatively. This provides us a way in preparing a long time surviving entangled quantum states needed in experiment [21, 22]. The degeneracy also increases the asymptotic value of the 
linear entropy. This means the coherence loss is increased by the degeneracy. Comparing to the non-degenerate case, the amplitude of local maximum and minimum of the linear entropy is suppressed due to the degeneracy. The more average photon number affects the cavity dissipation by increasing the asymptotic value of the linear entropy of the atom and the system and making them lose their coherence more rapidly than usual. The better the initial coherence property of the atom the larger the asymptotic value of the linear entropy, i.e. the larger the coherence loss.

\section{References}

[1] W H Zurek Phys. Today 44, 36 (1991).

[2] L Davidovich, M Brune, J M Raimond, and S Haroche, Phys. Rev. A 53, 1295 (1996).

[3] J G Peixoto and M C Nemes, Phys. Rev. A 59, 3918 (1999).

[4] L. M. Duan and G. C. Guo, Phys. Rev. A 57, 2399 (1998).

[5] J I Cirac, P Zoller, H J Kimble, and H Mabuchi, Phys. Rev. Lett. 78, 3321 (1996).

[6] T Pellizari, Phys. Rev. Lett. 79, 5242 (1997).

[7] J I Cirac and P Zoller, Phys. Rev. A 50, R2799 (1994); C C Gerry, Phys. Rev. A $53,2857(1996)$.

[8] L Davidovich, N Zagury, M Brune, J M Raimond and S Haroche, Phys. Rev. A 50, R895 (1994).

[9] J I Cirac, A S Parkins, Phys. Rev. A 50, R441(1994).

[10] S B Zheng and G C Guang, Phys. Rev. Lett. 85, 2392 (2001). 
[11] M Brune et al, Phys. Rev. Lett. 77, 4887(1996).

[12] L Zhou, H S Song, Y X Luo and C Li, Phys. Lett. A 284, 156 (2001)

[13] Reshetov V A 2000 Preprint physics/0002047.

[14] L Zhou, H S Song,Y Li, Chin. Phys. 10, 413 (2001).

[15] L Zhou, H S Song and Y X Luo, J. Opt. B 4, 103 (2002).

[16] L Zhou, H S Song, C Li and Y Q Guo, Chin. Phys. 12, 45 (2003).

[17] M Fleischhauer, M Dlukiin, Phys. Rev. Lett. 84, 5094 (2000).

[18] X X Yi, C Li and J C Su, Phys. Rev. A 62,013819 (2000).

[19] M Brune, J M Raimond, P Goy, L Davidovich and S Haroche, Phys. Lett. 59, $1899(1987)$.

[20] L Zhou, Doctor Dissertation (Dalian University of Technology, Dalian 2003).

[21] S J D Phoenix and S M Barnett, J. Mod. Opt. 40, 979 (1993).

[22] I K Kudryavtstev and P L Knight, J. Mod. Opt. 40, 1673 (1993); J I Cirac and P Zoller, Phys. Rev. A 50, R4887 (1994).

\section{Figure Captions:}

Fig.1 Linear entropy of atom as a function of $\Omega t, S_{d a}$ with degenerate atomic levels (solid line), $S_{a}$ with two levels atomic state (dotted line), where $e=f=1 / \sqrt{2}, \kappa / \Omega=$ $0.02,|\alpha|^{2}=0.5$.

Fig.2a Linear entropy of the system (atom+field) as a function of $\Omega t$, where $e=$ $f=1 / \sqrt{2}, \kappa / \Omega=0.01$,solid line with $|\alpha|^{2}=1$, dotted line with $|\alpha|^{2}=0.5$.

Fig.2b Linear entropy of the atom as a function of $\Omega t$, where $e=f=1 / \sqrt{2}$, $\kappa / \Omega=0.02$, solid line with $|\alpha|^{2}=1$, dotted line with $|\alpha|^{2}=0.5$. 
Fig.3 Linear entropy of atom as a function of $\Omega t$, Where $\kappa / \Omega=0.02,|\alpha|^{2}=0.5$, solid line with $e=f=1 / \sqrt{2}$, dotted line with $e=0.4$. 


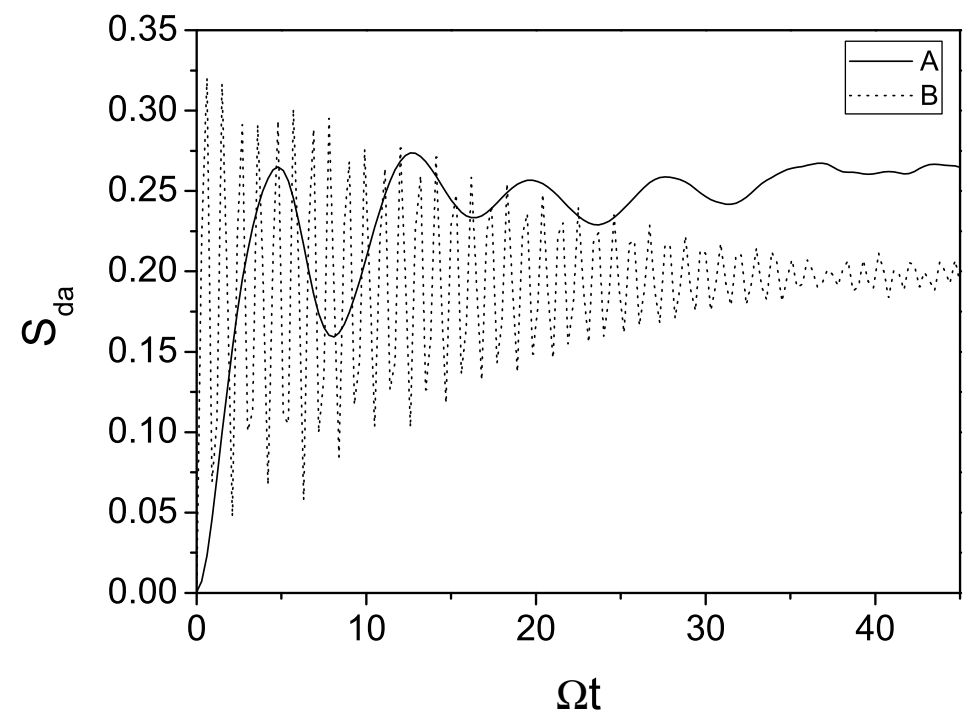




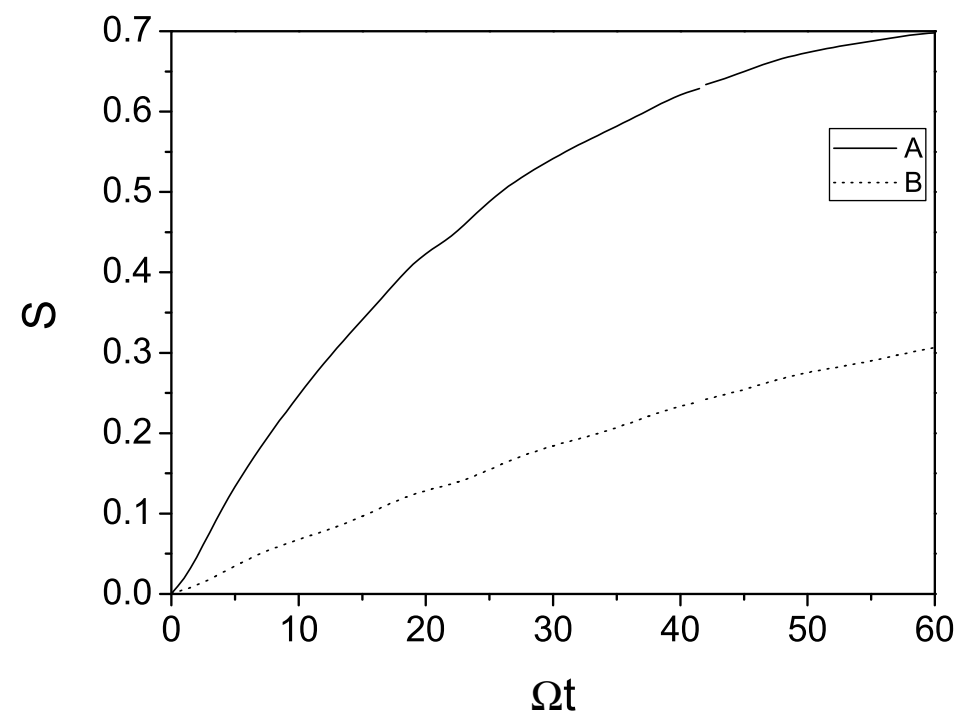




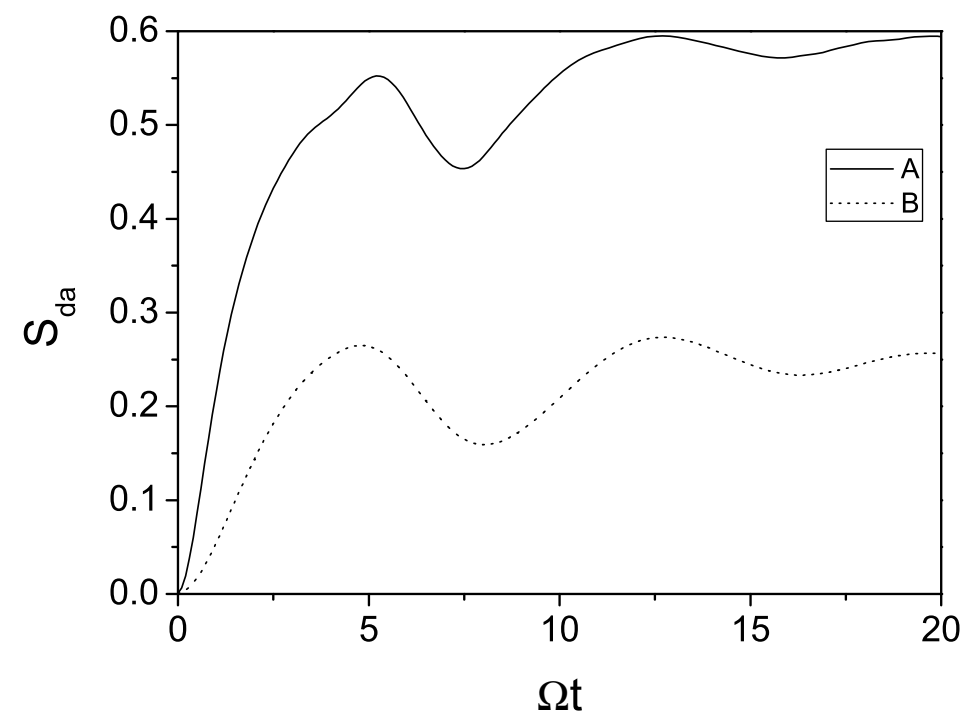




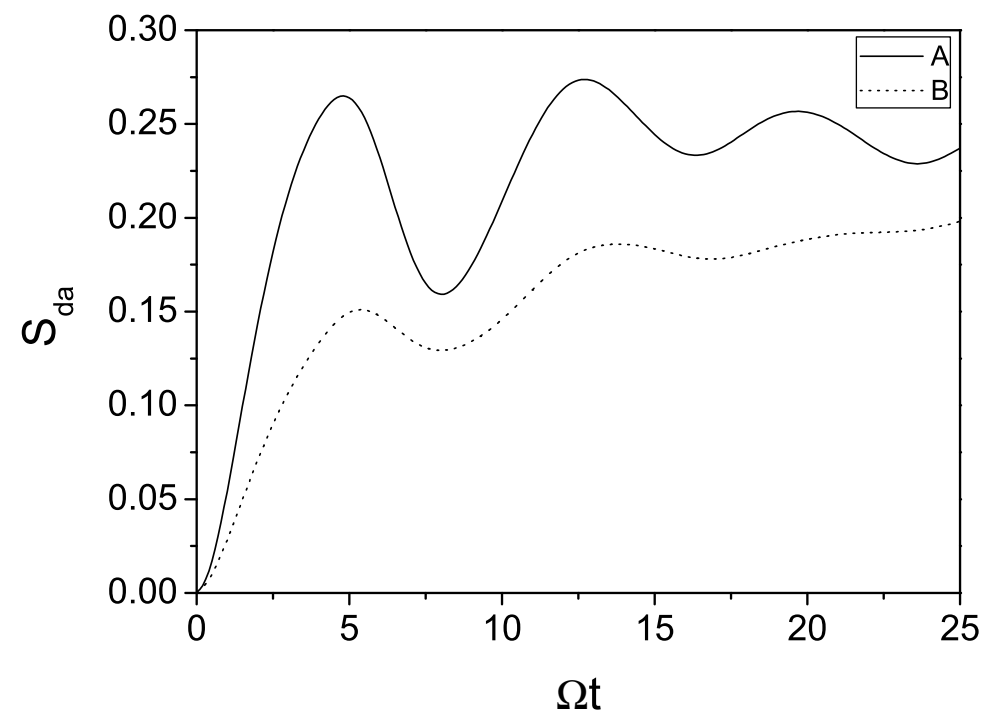

